\title{
The Effects of Knee Joint and Hip Abduction Angles on the Activation of Cervical and Abdominal Muscles during Bridging Exercises
}

\author{
Su-Kyoung Lee, PT, PhD ${ }^{1)}$, Du-Jin PARK, PT, MS²* \\ 1) Department of Physical Therapy, Gimhae College \\ 2) Department of Physical Therapy, Graduate School of Catholic University of Pusan: 9 Bugok 3-dong, \\ Geumjung-gu, Busan 609-757, Republic of Korea. TEL: +82 55-320-1735, FAX: +82 55-336-6222
}

\begin{abstract}
Purpose] The purpose of this study was to examine the effects of the flexion angle of the knee joint and the abduction angle of the hip joint on the activation of the cervical region and abdominal muscles. [Subjects] A total of 42 subjects were enrolled 9 males and 33 females. [Methods] The bridging exercise in this study was one form of exercise with a knee joint flexion angle of $90^{\circ}$. Based on this, a bridging exercise was conducted at the postures of abduction of the lower extremities at $0,5,10$, and $15^{\circ}$. [Result] The changes in the knee joint angle and the hip abduction angle exhibited statistically significant effects on the cervical erector spinae, adductor magnus, and gluteus medius muscles. The abduction angles did not result in statistically significant effects on the upper trapezium, erector spinae, external oblique, and rectus abdominis muscles. However, in relation to the knee joint angles, during the bridging exercise, statistically significant results were exhibited. [Conclusion] When patients with both cervical and back pain do a bridging exercise, widening the knee joint angle would reduce cervical and shoulder muscle activity through minimal levels of abduction, permitting trunk muscle strengthening with reduced cervical muscle activity. This method would be helpful for strengthening trunk muscles in a selective manner.

Key words: Bridging exercise, Knee and hip joint angle, Muscle activation
\end{abstract}

(This article was submitted Feb. 6, 2013, and was accepted Mar. 8, 2013)

\section{INTRODUCTION}

For spinal stabilization, the harmonious coordination of all of the trunk muscles is more important than the activation of specific muscles ${ }^{1}$. The spine is stabilized by the co-activation of the trunk muscles, and the spine muscles can be divided into global and local muscles. The global muscles are large muscles located on the body surface surrounding the abdominal and lumbar regions. These muscles mainly produce torque and are involved in overall trunk stability. Local muscles are intrinsic muscles located deep in the abdominal and lumbar regions. These muscles are directly connected to the spine and are therefore involved in the micro control and inter-segmental stabilization of the spine $^{2}$. Controlled cooperation between the global and local muscles maintains the state of spinal stabilization ${ }^{3,4)}$. Therefore, the co-contraction of the trunk muscles is necessary to maintain proper spinal stability in the treatment and prevention of lumbar pain ${ }^{1,3,5)}$.

A number of studies on the control of posture and movement have recently been conducted. In particular, various studies of core stability have attracted great interest $\left.{ }^{7}, 8\right)$. In light of this, bridging exercises have been used in trunk stabilization programs at clinics. Bridging exercises consist of postures in which patients with lumbar pain feel comfort-

*To whom correspondence should be addressed. E-mail: pt80dj@naver.com able and pain is decreased. In addition, the exercises can retrain the global and local muscles to coordinate with each other in a proper ratio ${ }^{4)}$. Bridging exercises also have an important relationship with functional movements, such as movements in bed, use of the toilet for patients, the removal of pressure, getting dressed using the lower extremities, and gait-related pelvic movements. Further, exercises include important motions that enable weight-bearing on the feet and the performance of the kneeling posture. They also increase the control of posture in moving from a sitting position to a standing position, and strengthen the extensor muscles of the lower spine and hip joints in preparation for the stance phase of gait $^{9}$. Moreover, the torque that influences the trunk in a state of pelvic stabilization is reported to be effectively delivered to the hip joints and lower extremities ${ }^{10)}$. Such stabilization of the trunk is necessary in order to increase spinal and pelvic stability during functional postures and movements, to strengthen related muscles and to recover control and balance of muscle movements ${ }^{6}$. Murphy et al. ${ }^{11)}$ reported on the relationship between cervical dysfunction and pelvic imbalance. They noted that the tension of the soft tissues that are stretched from the cervical region, such as a chain of muscles, skin, and fasciae, can create pelvic imbalance ${ }^{12}$. In addition, Lee and Lee ${ }^{13)}$ reported that during bridging exercises, the contraction of the abductors in the lower extremities and the activation of the abdominal muscles increased.

Kavcic et al. ${ }^{14)}$ conducted a study on muscle loading and 
spinal stability in which healthy adults performed lumba stabilization exercises. They reported that the abdominal curl, the side bridging exercise of the right isometric side support, the bridging exercise with the right leg lift, and the general bridging exercise had significantly high correlations with activation of the rectus abdominis muscle. However, during these bridging exercises, the activation of the abdominal muscles is considered to differ depending on the distance of the space between the feet that support the knee joint from the floor. In this respect, the present study attempted to identify the effects of the flexion angle of the knee joint and the abduction angle of the hip joint on the activation of the cervical region and abdominal muscles.

\section{SUBJECTS AND METHODS}

The subjects of this study were 45 healthy adults in their 20 's and 30's, both male and female, who understood and consented to participate and had the muscular strength, range of motion, and ability to balance that would enable them to perform the required exercises. Subjects with nervous and cardiopulmonary system problems or orthopedic musculoskeletal issues related to the trunk and lower extremities were excluded. A total of 42 subjects were selected, 9 males and 33 females. Their average age was $23.46 \pm$ 2.72 years, their average weight was $58.60 \pm 10.21 \mathrm{~kg}$, and their average height was $164.09 \pm 7.92 \mathrm{~cm}$. The bridging exercise in this study was one form of exercise with a knee joint flexion angle of $90^{\circ}$. The bridging exercise was conducted with the lower extremities abducted at $0,5,10$, and $15^{\circ}$. When performing the bridging exercise, the subjects crossed their arms and placed them on the chest. Then, the trunk and lower extremity were elevated to form a straight line, in which hip flexion was $0^{\circ}$. To prevent increase of lumbar lordosis during the bridging exercise, the exercise was practiced after learning the lumbar neutral position through the pelvic posterior tilting exercise. All trials were repeated three times and the trial order was randomized using a table of random sample numbers. Each exercise lasted for 5 seconds. The muscle activity data of the middle $3 \mathrm{sec}-$ onds, excluding the first and last one seconds, used for the analysis. To prevent fatigue during the exercise, there was a 1-minute break after each 5-second exercise. A surface EMG - MP150 WSW (BIOPAC system Inc. CA, USA) was used to record the subjects' muscle activities during the bridging exercise, and electrodes were attached to their cervical erector spinae, upper trapezius, external oblique, rec- tus abdominis, erector spinae, adductor magnus, and gluteus medius. The collected data were analyzed with SPSS for Windows (Ver. 18.0), and one-way analysis of variance (ANOVA) was employed to compare muscle activities of the lower limbs. As a post-hoc test, the LSD test was used to examine differences.

\section{RESULTS}

During the bridging exercise, the effects of the knee joint angle and the hip abduction angle on cervical and abdominal muscle activities were examined. The changes in the knee joint angle and the hip abduction angle exhibited statistically significant effects on the cervical erector spinae, adductor magnus, and gluteus medius muscles $(\mathrm{p}<0.05)$. The abduction angles did not result in statistically significant effects on the upper trapezius, erector spinae, external oblique, or rectus abdominis muscles $(p>0.05)$. However, the knee joint angles, during the bridging exercise exgibited, statistically significant effects on these muscles $(\mathrm{p}<0.05)$.

According to the results of the post-hoc tests, the cervical erector spinae muscle showed statistically significant changes in the bridging exercises at knee joint angles of $90^{\circ}$ and $60^{\circ}$, and at abduction angles of $0^{\circ}$ and $15^{\circ}(\mathrm{p}<0.05)$. The upper trapezius muscle exhibited statistically significant changes at a knee joint angle of $120^{\circ}$, and at all abduction angles $(\mathrm{p}<0.05)$. The abductor magnus muscle also showed statistically significant changes at a knee joint angle of $120^{\circ}$ and at all abduction angles $(\mathrm{p}<0.05)$ while the gluteus medius muscle showed statistically significant changes at a knee joint angle of $120^{\circ}$ and at abduction angles of $0^{\circ}, 10^{\circ}$, and $15^{\circ}(\mathrm{p}<0.05)$. At a knee joint angle of $90^{\circ}$, the gluteus medius muscle exhibited statistically significant changes at abduction angles of $0^{\circ}, 10^{\circ}$, and $15^{\circ}(\mathrm{p}<0.05)$. However, at a knee joint angle of $45^{\circ}$, it showed statistically significant changes at all abduction angles $(\mathrm{p}<0.05)$ (Tables $1-7)$.

\section{DISCUSSION}

The stabilization of the trunk is necessary to increase spinal and pelvic stability in functional postures and movements, to strengthen the relevant muscles, and to recover control and balance of muscles and movements ${ }^{6}$. . Abdominal muscle activity is an essential element in hip muscles, stabilization of the pelvis against pulling forces, and the forces on the trunk in state of pelvic stabilization are ef-

Table 1. Comparison of CE muscle activation among the various bridging exercise positions

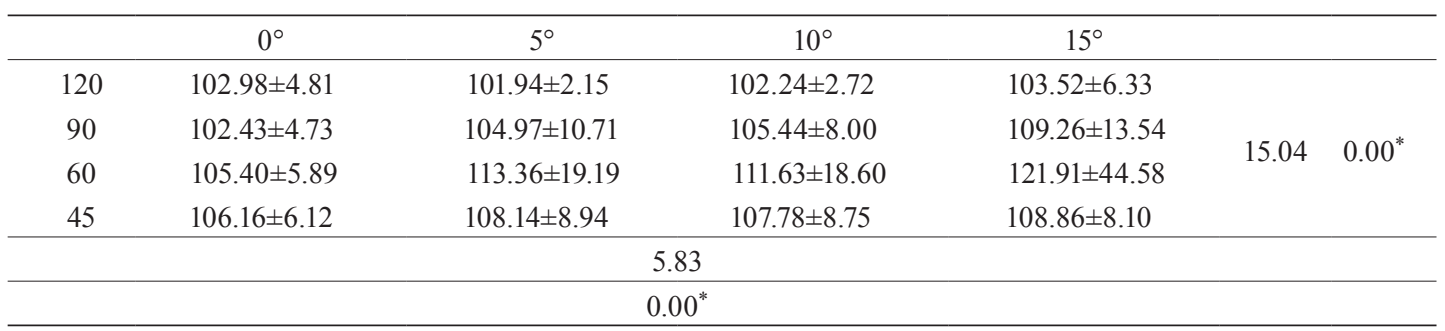

Unit: \%RVC, mean \pm SD, *: p $<0.05$, CE: Cervical Elector Spinae 
Table 2. Comparison of TU muscle activation among the various bridging exercise positions

\begin{tabular}{cccccc}
\hline \multicolumn{1}{c}{$0^{\circ}$} & $5^{\circ}$ & \multicolumn{1}{c}{$10^{\circ}$} & \multicolumn{1}{c}{$15^{\circ}$} \\
\hline 120 & $112.99 \pm 14.67$ & $107.63 \pm 10.94$ & $106.54 \pm 9.61$ & $107.48 \pm 8.00$ & \\
90 & $113.90 \pm 18.94$ & $115.29 \pm 23.81$ & $121.38 \pm 23.87$ & $119.39 \pm 21.33$ & \\
60 & $124.62 \pm 36.44$ & $152.72 \pm 75.67$ & $145.12 \pm 68.16$ & $169.18 \pm 101.82$ \\
45 & $155.96 \pm 78.01$ & $150.97 \pm 97.75$ & $153.11 \pm 95.88$ & $163.74 \pm 110.56$ \\
\hline \multicolumn{5}{c}{1.29} \\
\hline \multicolumn{5}{r}{0.28} \\
\hline
\end{tabular}

Unit: \%RVC, mean \pm SD, *: p<0.05, TU: Trapezius Upper Fiber

Table 3. Comparison of EO muscle activation among the various bridging exercise positions

\begin{tabular}{|c|c|c|c|c|}
\hline & $0^{\circ}$ & $5^{\circ}$ & $10^{\circ}$ & $15^{\circ}$ \\
\hline 120 & $102.44 \pm 2.90$ & $101.22 \pm 3.49$ & $101.40 \pm 3.67$ & $101.42 \pm 3.36$ \\
\hline 90 & $102.73 \pm 2.22$ & $102.43 \pm 3.64$ & $102.77 \pm 4.36$ & $103.86 \pm 6.16$ \\
\hline 60 & $104.05 \pm 3.33$ & $103.54 \pm 4.87$ & $104.20 \pm 5.54$ & $104.93 \pm 5.82$ \\
\hline 45 & $104.53 \pm 6.19$ & $104.05 \pm 5.12$ & $104.03 \pm 5.00$ & $104.22 \pm 5.03$ \\
\hline \multicolumn{5}{|c|}{1.01} \\
\hline \multicolumn{5}{|c|}{0.39} \\
\hline
\end{tabular}

Unnit: \%RVC, mean $\pm \mathrm{SD}, *$ : $<<0.05$, EO: External Oblique

Table 4. Comparison of RA muscle activation among the various bridging exercise positions

\begin{tabular}{cccccc}
\hline \multicolumn{1}{c}{$0^{\circ}$} & $5^{\circ}$ & $10^{\circ}$ & $15^{\circ}$ \\
\hline 120 & $103.79 \pm 3.60$ & $102.98 \pm 2.07$ & $103.20 \pm 1.92$ & $103.60 \pm 2.28$ & \\
90 & $104.06 \pm 2.63$ & $104.24 \pm 3.08$ & $104.53 \pm 2.74$ & $105.36 \pm 3.40$ & $*$ \\
60 & $106.43 \pm 3.99$ & $106.01 \pm 3.66$ & $106.02 \pm 3.02$ & $106.57 \pm 4.22$ \\
45 & $106.58 \pm 4.67$ & $107.33 \pm 4.54$ & $106.54 \pm 4.06$ & $107.04 \pm 4.03$ \\
\hline \multicolumn{5}{c}{0.92} \\
\hline \multicolumn{5}{c}{0.43} \\
\hline
\end{tabular}

Unit: $\%$ RVC, mean $\pm \mathrm{SD}, *$ : $\mathrm{p}<0.05$, RA: Rectus Abdominis

Table 5. Comparison of ES muscle activation among the various bridging exercise positions

\begin{tabular}{ccccc}
\hline & $0^{\circ}$ & $5^{\circ}$ & $10^{\circ}$ & $15^{\circ}$ \\
\hline 120 & $1420.53 \pm 1242.32$ & $1103.67 \pm 1197.76$ & $1319.80 \pm 1206.87$ & $1300.06 \pm 1159.79$ \\
90 & $1623.86 \pm 1311.32$ & $1633.84 \pm 1438.24$ & $1549.16 \pm 1326.70$ & $1575.55 \pm 1323.07$ \\
60 & $1987.64 \pm 1549.80$ & $2042.17 \pm 1643.34$ & $1934.93 \pm 1546.34$ & $2019.28 \pm 1643.39$ \\
45 & $2099.32 \pm 1661.83$ & $2443.00 \pm 1726.72$ & $2299.87 \pm 1726.72$ & $2405.60 \pm 1889.67$ \\
\hline \multicolumn{5}{c}{0.39} \\
\hline \multicolumn{5}{c}{0.99} \\
\hline
\end{tabular}

Unit: \%RVC, mean $\pm \mathrm{SD}, *$ : $\mathrm{p}<0.05$, ES: Erector Spinalis

Table 6. Comparison of AM muscle activation among the various bridging exercise positions

\begin{tabular}{cccccc}
\hline & $0^{\circ}$ & $5^{\circ}$ & $10^{\circ}$ & $15^{\circ}$ \\
\hline 120 & $113.28 \pm 23.25$ & $102.16 \pm 24.98$ & $100.03 \pm 20.71$ & $100.10 \pm 20.97$ & \\
90 & $120.82 \pm 15.85$ & $115.28 \pm 35.85$ & $114.40 \pm 32.67$ & $112.55 \pm 31.76$ & $*$ \\
60 & $137.62 \pm 44.14$ & $132.95 \pm 55.65$ & $129.02 \pm 53.17$ & $126.82 \pm 48.67$ & $*$ \\
45 & $137.14 \pm 53.30$ & $126.69 \pm 45.80$ & $123.91 \pm 41.11$ & $125.20 \pm 49.20$ \\
\hline \multicolumn{5}{c}{2.78} \\
\hline \multicolumn{5}{c}{$0.04^{*}$} \\
\hline
\end{tabular}

Unit: \%RVC, mean \pm SD, *: p<0.05, AM: Adductor Magnus 
Table 7. Comparison of GM muscle activation among the various bridging exercise positions

\begin{tabular}{ccccc}
\hline \multicolumn{5}{c}{ Hip abd } \\
\hline Kne flex & $0^{\circ}$ & $5^{\circ}$ & $10^{\circ}$ & $15^{\circ}$ \\
120 & $131.42 \pm 29.98$ & $141.90 \pm 39.40$ & $166.91 \pm 62.29$ & $178.10 \pm 58.11$ \\
90 & $156.09 \pm 43.14$ & $188.46 \pm 66.26$ & $191.32 \pm 59.42$ & $207.68 \pm 74.422^{*}$ \\
60 & $149.11 \pm 35.78$ & $170.10 \pm 48.18$ & $185.64 \pm 56.86$ & $198.07 \pm 75.98^{*}$ \\
45 & $141.70 \pm 40.42$ & $174.28 \pm 66.31$ & $178.41 \pm 61.46$ & $190.91 \pm 66.45$ \\
\hline \multicolumn{5}{c}{22.58} \\
\hline \multicolumn{5}{c}{$0.00^{*}$} \\
\hline
\end{tabular}

Unit: \%RVC, mean $\pm \mathrm{SD}, *$ : $\mathrm{p}<0.05$, GM: Gluteus Medius

fectively delivered to the hip joints and lower extremities ${ }^{10)}$. This study aimed to examine the influences of the knee joint angle and the hip abduction angle on the activation of the trunk, cervical, and abdominal muscles during a specific bridging exercise. To this end, electromyograms were recorded of seven muscles during bridging exercises with different according feet-to-hip length changes, i.e., changes of the knee joint angle with the hip in a neutral position without abduction of the lower extremities. The EMGs exhibited statistically significant changes in all the muscles. However, changes in muscle activity at different hip abduction angles during the bridging exercise showed statistically significant results only in the cervical erector spinae, adductor magnus, and gluteus medius muscles. These results indicate an increase in abdominal pressure due to the contraction of the abdominal muscles in accordance with changes in the knee joint angle. However, while abdominal muscle activity did not change during abduction, the muscles involved in pelvic abduction as well as the cervical and shoulder muscles, which served as a fixation point on the floor during the bridging exercise, exhibited increased activity.

Knowing the levels of trunk muscle activation during trunk stabilization exercises is important for controlling exercise intensity when designing and prescribing exercise programs $^{5)}$. In the present study, abduction of the hip joint increased the activation of the cervical and shoulder muscles rather than strengthening the trunk muscles; therefore, it would not be helpful for patients with both cervical and back pain. Lee ${ }^{15)}$ stated that when a patient complains of cervical pain, an increase in the knee joint angle can result in a corresponding decline in the muscle activity of the cervical region.

In conclusion, when patients with both cervical and back pain do a bridging exercise, widening the knee joint angle and reducing cervical and shoulder muscle activity through minimal levels of abduction, cervical muscle activity can be reduced and trunk muscle strength reinforced. This method would be helpful for strengthening trunk muscles in a selective manner.

\section{REFERENCES}

1) Stevens VK, Bouche KG, Mahiru NN, et al.: Trunk muscle activity in healthy subjects during bridging stabilization exercises. BMC Musculoskelet Disord, 2006, 7: 75 . [CrossRef]

2) Bergmark A: Stability of the lumbar spine: a study in mechanical engineering. Acta Orthop Scand Suppl, 1989, 230: 1-54. [Medline]

3) Marshall PW, Murphy BA: Core stability exercises on the off a swiss ball. Arch Phys Med Rehabil, 2005, 86: 242-249. [Medline] [CrossRef]

4) Stevens VK, Coorevits PL, Bouche KG, et al.: The influence of specific training on trunk muscle recruitment patterns in healthy subjects during stabilization exercises. Man Ther, 2007, 12: 271-279. [Medline] [CrossRef]

5) Lehman GJ, Hpda W, Oliver S: Trunk muscle activity during bridging exercise on and off a Swiss ball. Chiropr Osteopat, 2005, 13: 14-21. [Medline] [CrossRef]

6) Richardson CA, Snijders CJ, Hides JA, et al.: The relation between the transverses abdominis muscle, sacroiliac joint mechanics, and low back pain. Spine, 2002, 27: 399-405. [Medline] [CrossRef]

7) Akuthota V, Nadler SF: Core strengthening. Arch Phys Med Rehabil, 2004, 85: S86-S92. [Medline] [CrossRef]

8) Page P: Sensorimotor training: a "global" approach for balance training. J Bodyw Mov Ther, 2006, 10: 77-84. [CrossRef]

9) O'Sullivan SB, Schmitz TJ: Physical Rehabilitation: Assessment and treatment. 4th ed. Philadelphia: F.A. Davis, 2001, pp 245-255.

10) Neumann DA: Kinesiology of the Musculoskeletal System: Foundation for physical rehabilitation. 1st ed. St. Louis: Mosby, 2002, pp 214-231.

11) Murphy DR: Conservative Management of Cervical Spine Syn. McGrawHill Companies, 2000, pp 60-64; 607-613.

12) Lewit K: Manipulation Therapy in Rehabilitation of the Locomotor System. 2nd ed. Boston: Butterworth Heinemann, 1991.

13) Lee SY, Lee SK: The impact of abductor and adductor contraction in a bridging exercise on muscle activities in of the abdominal region and the lower extremities. J Phys Ther Sci, 2012, 24: 1095-1097.

14) Kavcic N, Grenier S, McGill SM: Quantifying tissue loads and spine stability while performing commonly prescribed low back stabilization exercises. Spine, 2004, 29: 2319-2329. [Medline] [CrossRef]

15) Lee SK, Moon DC, Cho HR: Effects of trunk and neck extensor muscle activity on the bridging exercise according to knee joint angle. J Phys Ther Sci, 2013, (in press) 